\title{
Basic Repairs and Maintenance for Home Landscape Irrigation Systems ${ }^{1}$
}

Mary S. McCready, Michael D. Dukes, and Kati Migliaccio²

\section{Introduction}

Many home owners use automated systems to irrigate their lawns and ornamental plants. In addition, nearly all newly built residential properties in Florida are irrigated using automatic systems with permanently installed sprinklers. While the systems are installed to provide minimal maintenance and automated irrigation, routine maintenance and repairs are required to correct component failure and damage.

Prompt repair of irrigation system problems prevents loss or damage to landscapes and wasted water. Identifying and correcting the specific problem can prevent additional harmful impacts. For example, dry areas in a landscape can appear to be lacking water, but the lack of water may be due to a broken sprinkler or some other problem causing a loss of pressure in the irrigation system. Simply setting the irrigation timer to a longer run time will not make up for dry areas occurring from leaks, clogs or broken irrigation pipe.
Some irrigation system problems are easily fixed, but they have to be identified to be repaired. The most important part of maintaining an irrigation system is conducting regular inspections while the system is operating. Some basic problems commonly encountered when checking irrigation systems are:

- Clogged sprinklers

- Leaking sprinklers and valves

- Obstructed sprinklers

- Overspray onto sidewalks, streets or buildings

- Design problems

- Missing nozzles

- Pipe leaks or breaks

Some of these problems are easily repaired while some may require the help of an irrigation contractor.

1. This document is AE451, one of a series of the Agricultural and Biological Engineering Department, Florida Cooperative Extension Service, Institute of Food and Agricultural Sciences, University of Florida. Original publication date September 2009. Revised October 2009. Visit the EDIS Web site at http://edis.ifas.ufl.edu.

2. Mary S. McCready, extension agent, Miami-Dade County Extension; Michael D. Dukes, associate professor, Department of Agricultural and Biological Engineering; Kati Migliaccio, assistant professor, Department of Agricultural and Biological Engineering, Tropical Research and Education Center-Homestead FL; Florida Cooperative Extension Service, Institute of Food and Agricultural Sciences, University of Florida, Gainesville, FL 32611.

The Institute of Food and Agricultural Sciences (IFAS) is an Equal Opportunity Institution authorized to provide research, educational information and other services only to individuals and institutions that function with non-discrimination with respect to race, creed, color, religion, age, disability, sex, sexual orientation, marital status, national origin, political opinions or affiliations. U.S. Department of Agriculture, Cooperative Extension Service, University of Florida, IFAS, Florida A. \& M. University Cooperative Extension Program, and Boards of County Commissioners Cooperating. 0 Lar ) HUHUKDQF $\square, Q M M P \square$ HDQ 


\section{Clogged Sprinklers}

Sprinklers become clogged over time by dirt and debris that is trapped in the filter or nozzle of the sprinkler. When a sprinkler is clogged, little or no water will be able to exit the nozzle while the system is running (Figure 1). Two of the main types of sprinklers are sprays and rotors. For more information on the types of sprinklers used for irrigation in the residential landscape see CIR825:

Irrigation of Lawns and Gardens http://edis.ifas.ufl.edu/WI003.

\section{Sprays}

- Raise and secure the pop-up part (riser) of the sprinkler using a helper or a soft surfaced clamping device. Use minimal force to not damage the riser.

- Unscrew the top part of the spray head (turning counter clockwise), while water is turned off, and remove the filter from the inside of the sprinkler body (Figure 2). Note: To remove the filter from the body of the sprinkler you may need tweezers or a paper clip.

- Rinse filter and the spray nozzle off thoroughly.

- While the spray is dismantled, the irrigation line should be flushed to ensure no other debris is in the system.

- Replace the filter and the nozzle making sure that the spray of water is aimed in the proper direction. Turn on the irrigation and ensure that water spray is reaching the targeted area.

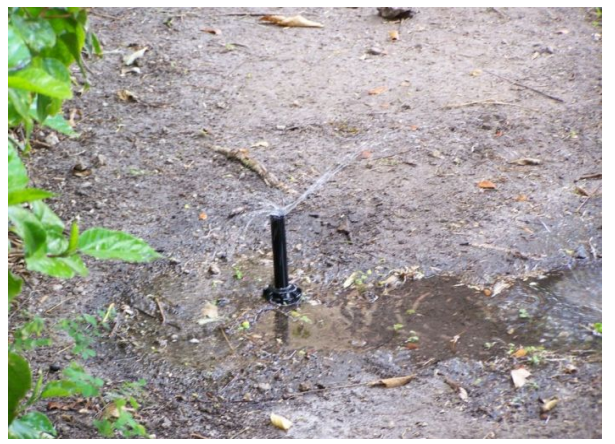

Figure 1. Picture of a clogged spray type sprinkler. Credits: Michael Gutierrez

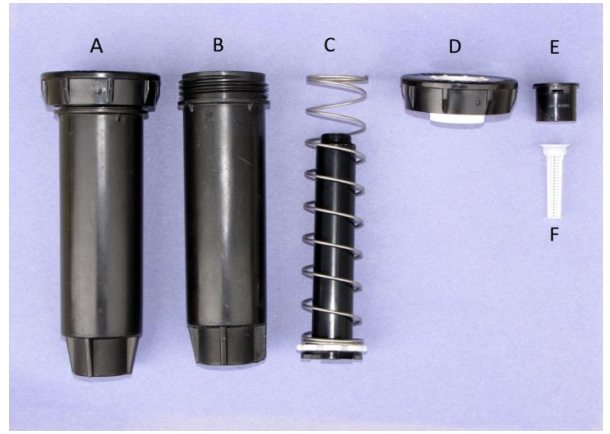

Figure 2. The components in a spray type sprinkler. The assembled spray is shown (A) along with the unassembled spray ( $B$ through $F)$. The unassembled parts include the body (B), the spring and pop-up portion (C), the nozzle (E) and the filter $(F)$. Credits: Bernard Cardenas-Lailhacar

\section{Rotors}

- The internals of the rotor need to be removed because the small basket-shaped filter is at the bottom of the rotor body. This can usually be performed by unscrewing the top portion of the rotor from the body using counter clockwise turns.

- Remove the filter.

- Rinse filter thoroughly.

-While the rotor is dismantled, the irrigation line should be flushed to ensure no other debris is in the system. Use care that dirty water and trash doesn't drain back into the open rotor body.

- Replace filter and nozzle.

- Turn on the irrigation and adjust the rotor's coverage pattern if necessary.

\section{Sprinkler and Valve Leaks}

Leaks can occur in sprinklers when seals become worn over time (Figure 3 and Figure 4). Often, leaking sprinklers are a result of damage that occurred during mowing or other lawn maintenance. Automobiles that accidentally drive over sprinkler heads can also cause large leaks. Leaks in the irrigation system can lead to significant loss of pressure, which causes dry areas in the landscape. Unchecked water loss results in high water bills and overly wet areas in the landscape near the leak, which can lead to disease and the presence of weeds. 
The location of a leak determines how to repair it.

- Some types of rotors allow for replacement of the seal inside the sprinkler. In general, once the sprinkler body (both sprays and rotors) has a large leak the entire sprinkler should be replaced.

- Many models of sprinklers are available at your local hardware store. Care should be taken to ensure that replacement sprinklers have the same operating characteristics (pressure, throw diameter, arc, etc.) as the damaged sprinkler. Sprinklers can be replaced easily by unscrewing the old sprinkler and replacing it with a new one.

- If the sprinkler body is located below ground level, the area around it should be dug out so as to free the body of the sprinkler from the soil. Clean the area where the new sprinkler will screw on so that soil will not be introduced into the system when the sprinklers are changed. Leaks often develop from broken pipe connections at the base of sprinklers, especially near streets and driveways. Flexible connection kits are available to reduce the occurrence of this breakage. Use a hand shovel when digging around the sprinkler to prevent cutting the irrigation line running to the sprinkler. Note: When digging, look out for buried irrigation control cable which is sometimes installed in the same ditch alongside the piping. It can be easily cut with a shovel making an additional repair necessary.

Leaks can develop in irrigation valves, whether from high pressure in the system or from general wear and tear (Figure 5 and Figure 6). For electric valves, wire connections should be made using waterproof wire connectors (also called grease caps) to prevent the loss of signal between the irrigation timer and the valve due to water damage (especially since the valves are typically buried underground as in Figure 5).

There are several types of irrigation valves, each having different problems and maintenance requirements. The problems that can cause leaks in valves depend on the type of valve. In general, if the valve has an external crack it will need to be replaced. Many irrigation equipment suppliers stock solenoids, gaskets and o-rings to repair valves. For leaking valves, a local irrigation contractor can assist with the repair. More information on irrigation valves is provided in CIR825: Irrigation of Lawns and Gardens http://edis.ifas.ufl.edu/WI003.

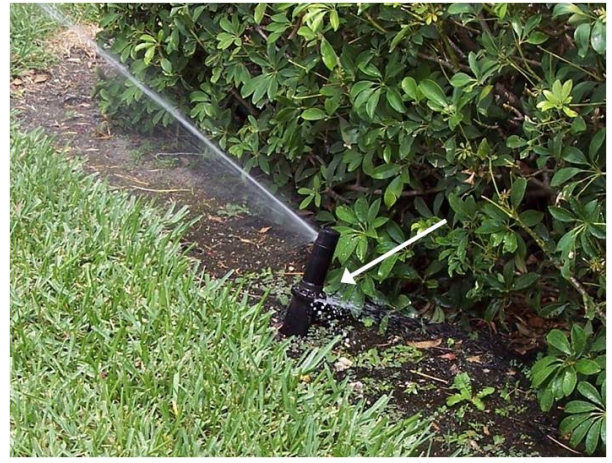

Figure 3. Leak in a rotor due to a worn seal indicated by the arrow. Note that the rotor body is crooked and should be buried to maintain vertical alignment and prevent damage from mowing. Credits: Ernesto Clark

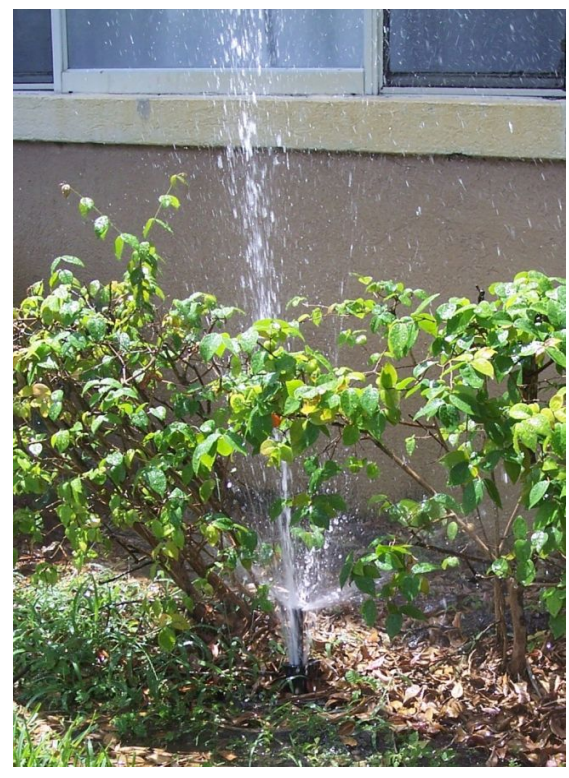

Figure 4. Leak in a spray due to a worn seal. Credits: Ernesto Clark

\section{Obstructed Sprinklers}

It is easy to forget the location of pop-up sprinklers in the landscape depending on the type of plants surrounding them. Make sure that objects, such as trash cans and plants are not placed in a location where they obstruct a sprinkler (especially when deciding where new plantings will be located). If something is located in front of the sprinkler, the irrigation will not reach its designated path and a dry spot will form in the landscape. 


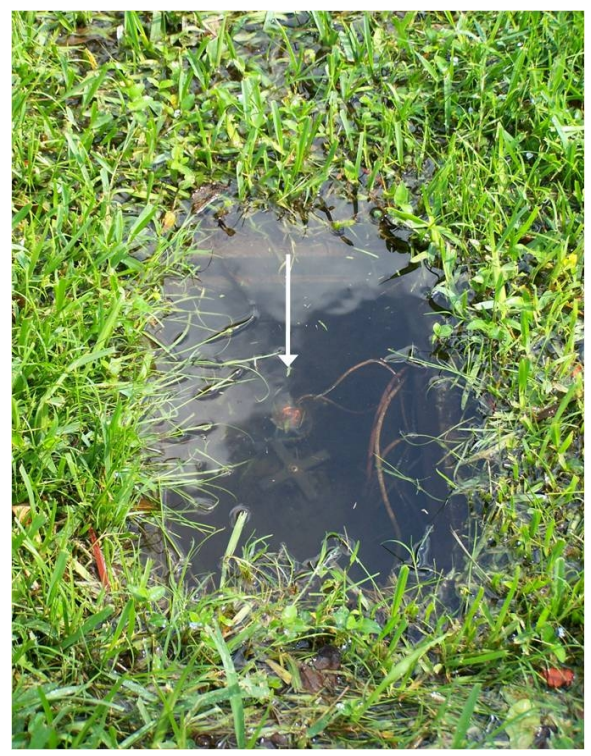

Figure 5. Leaking solenoid valve (indicated by arrow) covered in water after irrigation. Credits: Michael Gutierrez

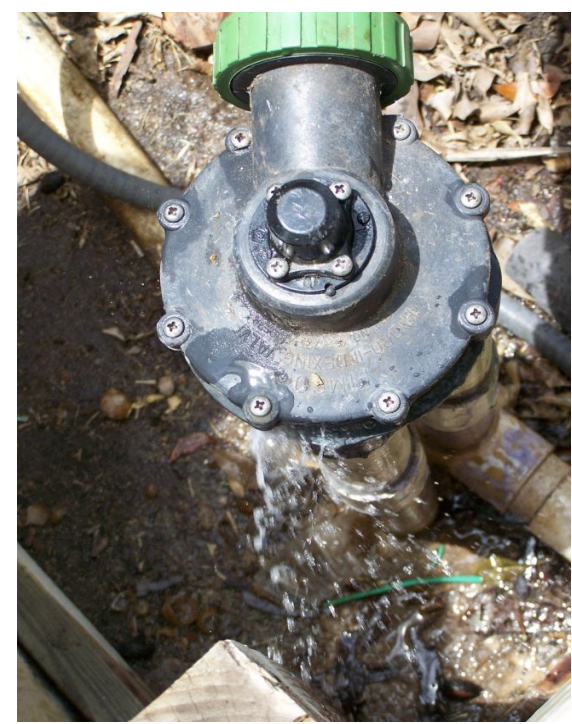

Figure 6. Leak in an indexing valve during irrigation due to high pressure in the system. Credits: Michael Gutierrez

It is also important to maintain the area surrounding a sprinkler to prevent obstruction of the spray. Turfgrass can grow over pop-up sprinklers making it impossible for the sprinklers to pop-up. Shrubs and overhanging tree branches may also need to be pruned back far enough to keep the sprinkler from being obstructed. Over time, sprinklers can be pushed further into the ground causing the spray of the sprinkler to not reach surrounding areas. This can happen from vehicles, including lawn mowers, being driven over the sprinkler (Figure 7).

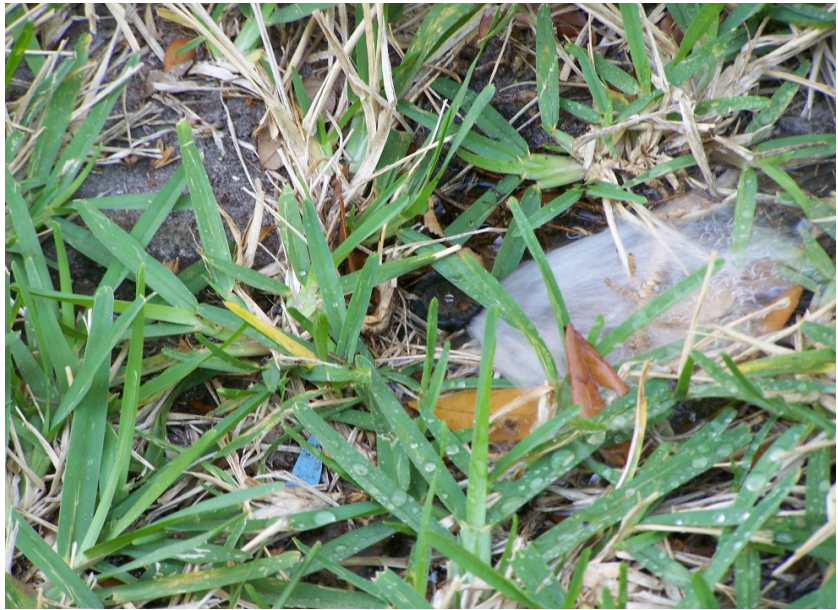

Figure 7. Spray head that no longer pops-up causing irrigation to only reach turfgrass within a couple of inches of the spray. Credits: Michael Gutierrez

\section{Overspray}

Sprinklers require adjustment to ensure water is properly applied to the right areas. If a street or house is wet after an irrigation cycle, the irrigation system should be evaluated and modified so that water is applied only to the landscape (Figure 8).

A common problem is incorrectly positioned sprinklers. They can be slightly tilted so that the irrigation is not directed towards the target area (Figure 9). To repair this type of problem, remove soil away from the side of the sprinkler, straighten the sprinkler and then replace and pack the soil around the sprinkler body.

Sometimes poor irrigation system design causes problems (Figure 10). The length of the spray of a sprinkler should be taken into account when designing a system. If spray or rotor type sprinklers are spraying outside of the landscaped area and the length of spray cannot be adjusted, they should be replaced with different sprinklers which have more appropriate application radius. The application radius is also affected by the pressure in the system. Sprinklers should be spaced so that the edge of the pattern from any sprinkler in the zone will touch its neighboring sprinkler - called "head to head spacing." This will enhance uniform coverage and reduce the occurrence of dry spots. More information on the uniformity of irrigation coverage can be found in AE43: Understanding the Concepts of Uniformity and Efficiency in Irrigation http://edis.ifas.ufl.edu/AE364. 
The arc and/or radius of some sprinklers are adjustable and should be checked regularly to make sure they are irrigating the proper area. Modifying the arc and/or the radius of a sprinkler can be a simple repair.

\section{Rotors}

- Both the radius and the arc of spray can be adjusted on most rotors. The nozzle, the part of the rotor where the water stream exits the rotor, is interchangeable within brands and specific model rotors to modify the flow rate and distance of throw or radius. Small radius changes can be adjusted for, but larger changes are made by changing the nozzle opening size. Several nozzle sizes are available from the manufacturer for each rotor. Note: Some rotors can be adjusted using either a flat-bladed screwdriver and others can be adjusted using a tool provided by the manufacturer. Read manufacturer instructions before attempting to adjust rotors.

\section{Sprays}

- The radius, or distance water is thrown, can be adjusted on most sprayers by turning the small screw at the top of the nozzle. Turning the screw clockwise will reduce the radius; turning the screw counter clockwise will increase the radius (within the nozzle`s capacity).

- For some spray type sprinklers the arc can be adjusted, but not all. Manufacturer instructions provide information on the adjustability of the spray heads. Sprayer nozzles are easily exchanged to obtain the desired pattern - and may have been replaced with an improper pattern. For example, a 360 degree pattern may have been installed next to a building or sidewalk where a 45, 90, or 180 degree pattern would be correct. Note: Not all manufacturers ${ }^{2}$ nozzles are interchangeable.

The spray direction may be twisted out of alignment with the landscaping over time and need to be repositioned. Note: Twist the pop-up portion of the spray head, while the irrigation is turned on, until the spray is directed toward the landscape.

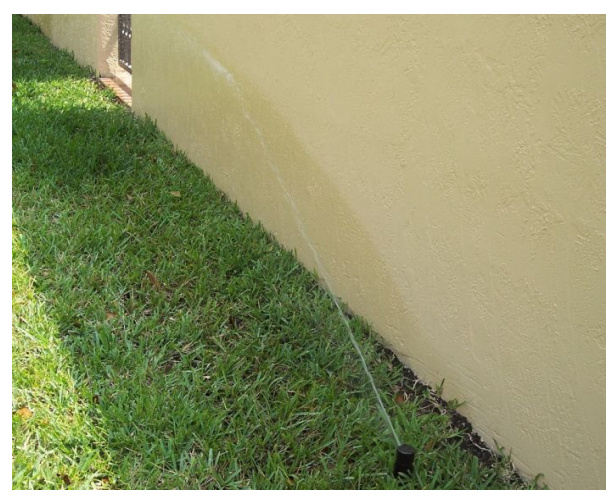

Figure 8. Rotor spraying water on the side of a house instead of the landscape. Credits: Mary McCready

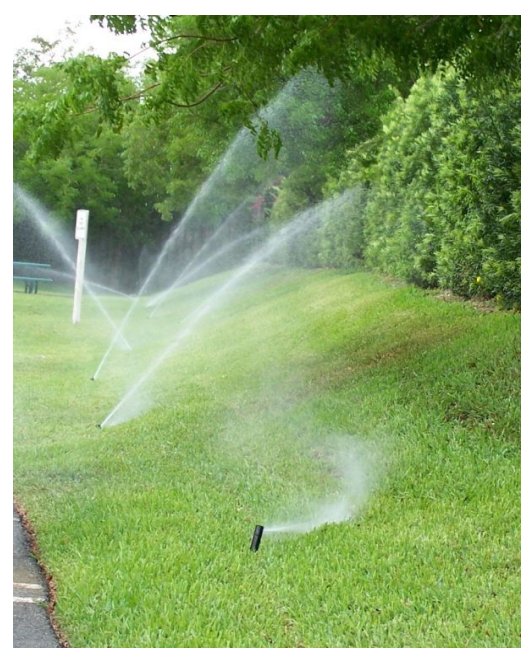

Figure 9. Tilted rotor spraying directly into turfgrass. Credits: Michael Gutierrez

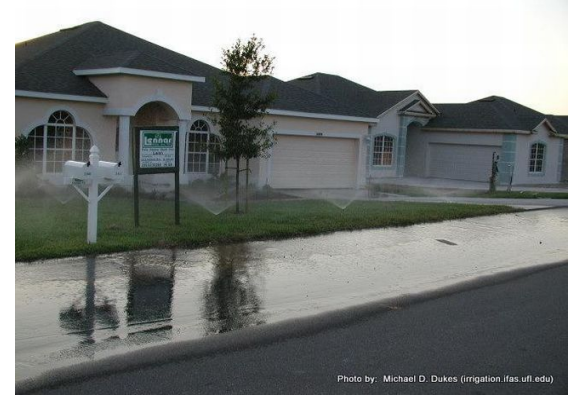

Figure 10. Wet area on pavement around landscaping due to poorly designed irrigation. Credits: Michael D. Dukes

\section{Missing Nozzles}

Nozzles from spray type irrigation systems can become detached from the sprinkler body over time or cut off by mowers. When a nozzle is missing, the pressure to the rest of the system will be decreased, causing poor coverage and possibly dry areas. The area directly around the sprinkler will be wetter than 
usual, possibly leading to weed growth and or fungus (Figure 11). Replacement nozzles for many types of spray heads are available at local hardware stores or can be ordered through a local irrigation supply company.

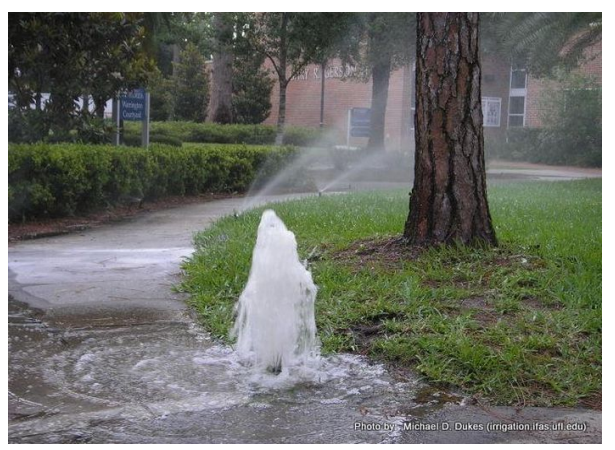

Figure 11. Nozzle on the sprinkler has been knocked off or broken. Credits: Michael D. Dukes

\section{Design Problems}

Landscaped areas can change over time and it is important to take into account the irrigation system design when modifying the landscape. For example, in Figure 12 the rotor is located behind a large shrub causing water to pool right next to the sprinkler. This system could be modified to have a spray type sprinkler on a riser in order to irrigate the entire area or more preferably microirrigation could be used to irrigate the shrub areas (Figure 13). For major design changes to your sprinkler system, contact an irrigation contractor.

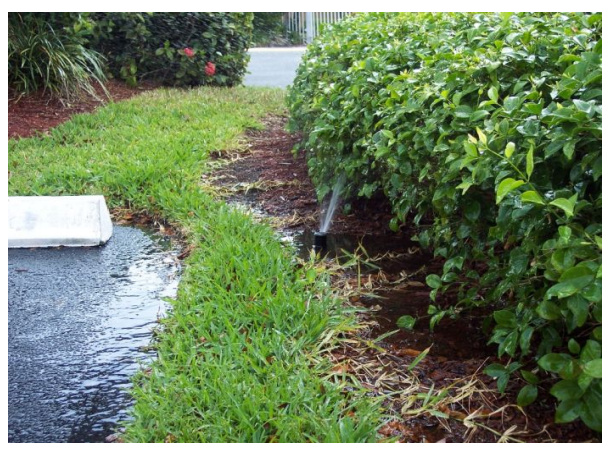

Figure 12. Irrigation system was designed before plants were installed. Modify system to meet plant needs. For established shrubs this sprinkler may not even be necessary. Credits: Mary McCready

\section{Pipe Leaks}

It is important to check for pipe leaks while the irrigation system is operating. These will likely be

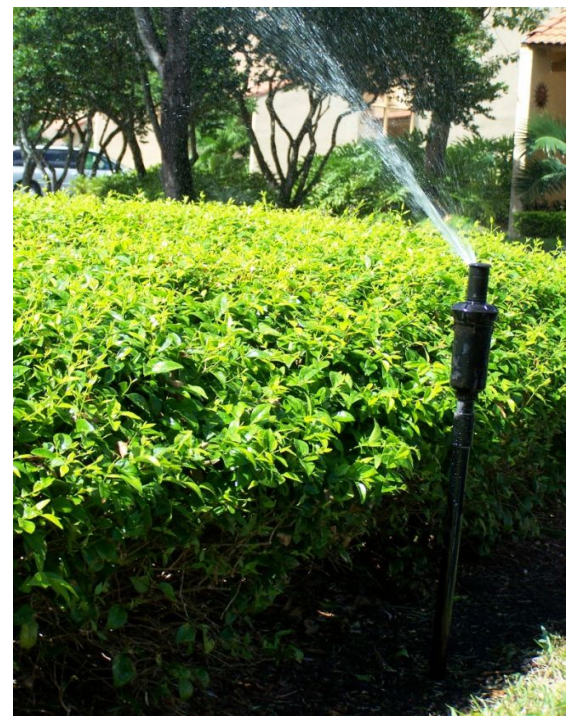

Figure 13. Riser added below rotor to give additional height so irrigation covers entire area. Credits: Michael Gutierrez

buried underground and may result in visible pools of water or large wet areas. If no wet area appears, but the zone has low pressure, look for an area of grass that is significantly greener than the rest and that may be where the system is leaking. Larger leaks may cause water to be observable above the soil surface (Figure 14). For large leaks that are below ground and require digging, a local irrigation contractor can be contacted. Most residential irrigation systems use white, polyvinylchloride (PVC) pipe material that is relatively easy to work with. The difficult part of the repair is exposing and cleaning the damaged area and keeping the area to be repaired clean while making the repair.

- Dig the soil away from the area with the leak unless the pipe leak is above ground (Figure 15). Note: Be careful not to cut other pipes.

- Cut the pipe. Note: For larger pipe leaks below ground, cut the pipe 4-5 inches from both sides of the break. For smaller pipe leaks cut away portion of pipe with the leak.

- Clean the pipe ends of any dirt that collects on the pipe. Pipe and couplings must be clean and dry for repair to work.

- Add PVC primer to the pipe ends and also primer to the inside of two straight couplers. 
- Apply a light coating of PVC pipe cement to the ends of the pipe and the inside of the straight couplers (only on the end that is going to be immediately attached to the pipe).

- Push couplers onto the ends of the PVC pipe until the coupler will not go on any farther.

- Cut a piece of PVC to fit the length between the two couplers, this length needs to account for the space between the two couplers and the length of pipe that fits into each coupler. Note: Make sure that the cut piece of pipe fits by inserting into the couplers before adding glue.

- Add PVC primer and pipe cement to one end of the pipe and one coupler and then push the pipe into the coupler.

- Add PVC primer and pipe cement to the other end of the cut PVC and the last coupler and push the pipe into the coupler. Note: Before turning irrigation on make sure the pipe cement has dried thoroughly (approximately 1 hour). Follow label instructions. When working with PVC pipe cement make sure to wear gloves.

- Special, extending type repair couplings are available that can make the repair job easier. Check your hardware, home improvement or irrigation supply store for availability and details.

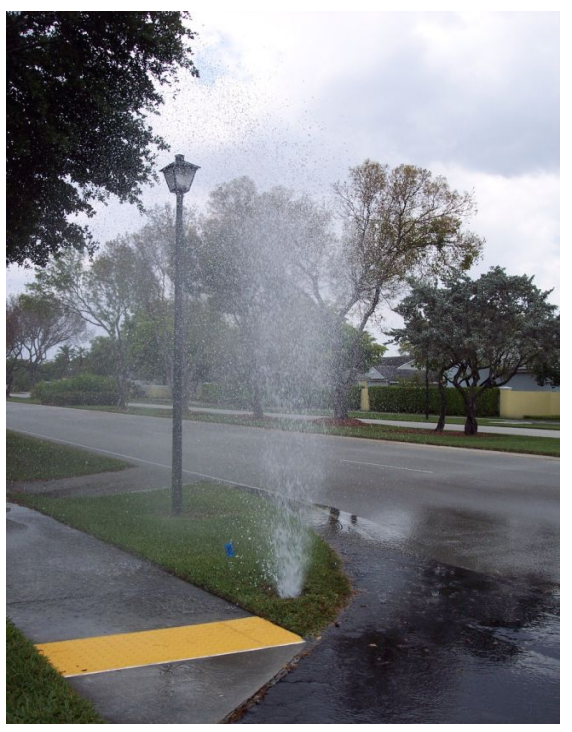

Figure 14. Large dramatic pipe leak. Credits: Ernesto Clark

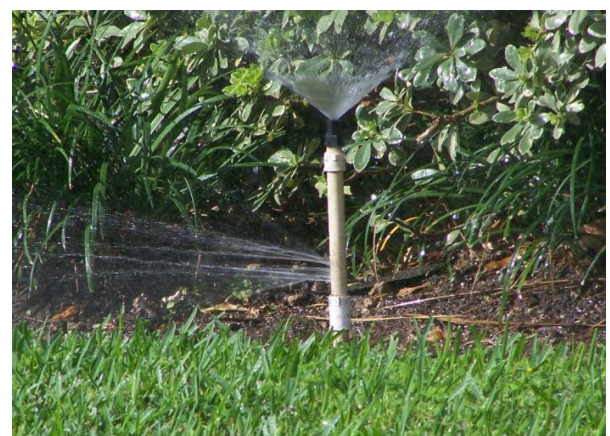

Figure 15. Pipe leak located below a spray head. Credits: Michael Gutierrez 